\title{
desarrollo ternolágiro de la industria del cemento
}

\section{El problema del polvo}

\section{Captación del polvo}

Las fábricas de cemento se han caracterizado durante mucho tiempo por el familiar penacho de polvo que salía por la chimenea. Pero algunas cosas están cambiando y el penacho va desapareciendo. Las razones son varias. Los habitantes de las zonas limítrofes con las fábricas de cemento no soportan el polvo y quieren disfrutar de una atmósfera pura. Los fabricantes se han dado cuenta de que el polvo no es otra cosa sino materia prima que ha sufrido parte del proceso: está completamente homogeneizada, finamente molida, exenta parcialmente de anhídrido carbónico e, inicialmente, contiene una considerable cantidad de calor. También se han dado cuenta que una acumulación de polvo en la fábrica representa un peligro para la salud, para la moral y para la vida de los propios empleados de la misma. Se puede ahorrar tiempo disminuyendo los incidentes o las molestias, se evitan complicaciones judiciales y se puede dar una prueba de buena voluntad. Han comprendido asimismo que la recuperación de polvos es un buen negocio y tiene como consecuencia el de un mayor orden en la fábrica.

Son muchos los métodos utilizados para recuperar el polvo. El más antiguo-tal vez-es el constituido por una cámara colectora, en donde los gases del horno pierden velocidad y su flujo cambia de sentido mediante unos deflectores. Son más eficaces los diversos tipos de recuperadores de ciclón y los filtros de mangas. Mejores resultados se han obtenido con la utilización de los precipitadores electrostáticos. Pero cuando parece haberse alcanzado casi la perfección, los habitantes de las zonas limítrofes trataron de pretender, no sólo la reducción del contenido de polvo en el aire, sino su total eliminación. En la isla de Oahu (Hawai) no se autorizó el montaje de una fábrica de cemento hasta que la empresa aseguró que ni se contaminaría en absoluto la atmósfera ni estropearía una fruta, denominada 'Waikiki, que supone muchos millones de dólares para la referida isla.

La eliminación completa del polvo se obtiene virtualmente con filtros de mangas de lana de vidrio, normalmente instalados a la salida de un ciclón. Este último capta la porción de polvo más gruesa, que tiene un bajo contenido en álcalis, y se envía inmediatamente al horno. La porción fina se descarga dentro de los filtros mediante un conducto de descarga. Válvulas de admisión controladas termostáticamente introducen aire auxiliar en los gases cargados de polvo, por lo cual la temperatura se reduce a unos $300^{\circ} \mathrm{C}$ cuando el polvo llega a las mangas del filtro.

La Ideal Cement Company ha construido recientemente una fábrica de cemento en Tijeras, Nuevo Méjico, con un solo horno (de 3,5 $\times 114 \mathrm{~m}$ ) para una producción anual de algo más de 200.000 t. Los polvos finos son captados por 1.280 mangas de tejido de vidrio impregnado de siliconas, cada una de unos $29 \mathrm{~cm}$ de diámetro y $7,5 \mathrm{~m}$ dẹ altura, colocadas en diez cámaras de 120 mangas cada una. La limpieza de las mangas se efectúa invirtiendo la presión sobre el 
tejido. Cada una de las cámaras se limpia automáticamente en ciclos periódicos. Apenas se ha descargado (por contrapresión), su polvo cae al fondo y se elimina por medio de un tornillo sinfín.

\section{Retorno de los polvos}

Cualquiera que sea el procedimiento utilizado para captar los polvos, una gran proporción de los álcaiis contenidos en los mismos se concentran en las fracciones más finas. Puesto que las normas del cemento limitan el contenido en álcalis, será necesario eliminar los polvos alcalinos $o$, en su defecto, depurar los polvos para eliminar los álcalis antes de retornarlos al horno. Existen varios procedimientos para realizar este proceso.

En los hornos que trabajan por vía húmeda, los polvos recuperados pueden tener tendencia a producir anillos de pasta debido a sus propiedades aglomerantes. Quizás se puede obviar este inconveniente mezclando adecuadamente el polvo con la pasta en el mezclador, bien con un sistema de alimentación por remolino, bien mediante la adición de sustancias inhibidoras, para impedir la aglomeración, o bien reamasando el polvo con la pasta o introduciéndola a la salida del recuperador de cadenas del horno. A veces el polvo se reamasa con agua en un pequeño molino y se reintroduce en el sistema de alimentación y dosificación. En las instalaciones por vía seca, el polvo puede reintroducirse directamente en el horno, añadiéndolo en la alimentación normal, o bien enviándolo a los homogeneizadores.

En todos los casos es importante que la recuperación de polvo se realice a ritmo continuo en vez de intermitentemente. La razón de todo esto estriba en que la composición de los polvos y sus necesidades de agua están muy alterados con relación a la de la mezela cruda y, por consiguiente, puede dar lugar a dificultades en la conducción del horno.

La recuperación de los polvos por la tobera del combustible se ha hecho con éxito en otras fábricas de cemento Mooser, de la Holderbank Company. El polvo puede introducirse directamente en la llama, o puede ser de antemano corregido en composición o mezclado con un aglomerante para impedir que partículas tan finas puedan ser transformadas por los gases de combustión. El polvo puede introducirse con el combustible por el conducto del aire primario o por medio de un conducto especial. En una nueva aplicación del procedimiento, Mooser ha encontrado que es posible, y quizás ventajoso y económico, inyectar otras sustancias en la llama junto con el combustible. Estas sustancias pueden ser escoria de alto horno, polvo de carbón y erudo molido.

\section{La eliminación de los álealis}

En algunos casos se ha encontrado que la arena, utilizada como inerte en el hormigón, reacciona con los álcalis del cemento provocando expansiones. Cuando se tenga que utilizar cemento con inertes reactivos, la norma puede limitar el contenido de álcalis del cemento a $0,6 \%$. Algunas fábricas de cemento pueden ajustarse a esta cifra sin ninguna dificultad, pero otras sólo pueden hacerlo instalando sistemas para la reducción y eliminación de los álcalis.

Es un hecho conocido que gran parte de los álcalis se eliminan del crudo por volatilización durante la cocción, y que la cantidad eliminada aumenta con Ia temperatura y con el tiempo de cocción. Sin embargo, la eliminación de los álcalis basada en el aumento de la temperatura y en el tiempo de cocción es muy costosa. Se sabe también que el cloruro cálcico mezclado con la alimentación o con el combustible puede rebajar considerablemente el contenido de álcalis. Los hornos que funcionan con carbón contienen más álcailis en el clínker que aquellos que emplean gas o nafta, como consecuencia de las combinaciones de los álcalis vaporizados con las cenizas. Y los hornos por vía húmeda normalmente contienen más álcalis en el clínker que los de vía seca, debido a su mayor longitud y a la menor temperatura de los gases de escape. 
Se ha visto también que la sosa se elimina más difícilmente que la potasa. La relación, que ha sido estudiada por Draper recogiendo datos de 73 fábricas de cemento, es:

$$
\mathrm{V}_{\mathrm{Na}_{2} \mathrm{O}}=\frac{\mathrm{V}_{\mathrm{K}_{2} \mathrm{O}-30}}{1,5}
$$

siendo: $V_{\mathrm{Na}_{2} \mathrm{O}}$ y $V_{\mathrm{K}_{z} \mathrm{O}}$ los porcentajes volatilizados de sosa y potasa, respectivamente. La ecuación indica que no se produce ninguna volatilización de sosa si no se elimina el $30 \%$ de potasa, y que, además, el punto de volatilización de la potasa será un $50 \%$ mayor que el de la sosa. La razón de esto es que los álcalis en el clínker están únicamente en forma de sulfatos, y que el sulfato sódico tiene una tensión de vapor más baja $(0,01 \mathrm{~mm} \mathrm{Hg})$ que el sulfato potásico $(0,4 \mathrm{~mm} \mathrm{Hg})$ a alta temperatura $\left(1.130^{\circ} \mathrm{C}\right)$.

Otros métodos similares se han experimentado para la eliminación de los álcalis, pero con escaso éxito. Se ha observado que adicionando ácido clorhídrico a la pasta se tiene un mejor control, a un coste más bajo, que empleando cloruro cálcico.

La Fuller Company ha hecho experimentos con el método «could-surface» (superficie fría) para la eliminación de los álcalis. Este método consiste en hacer pasar los gases sobre una superficie fría, formada por un conjunto de tubos de $5 \mathrm{~cm}$ de diámetro, refrigerados por agua. Se depositan dos clases de materiales: 1) A todo lo largo de los tubos, una incrustación blanca que contiene hasta un $60 \%$ de álcalis debida a la condensación; y 2) En la parte inferior de los tubos, un polvo oscuro que contiene un $15 \%$ de álcalis y que se atribuye a depósito por choque del polvo. A intervalos de cinco minutos los depósitos crecen hasta $1 \mathrm{~cm}$ de espesor. La materia oscura depositada en los tubos durante el enfriamiento y el depósito blanco pueden rasparse o disolverse en agua.

Aunque los álcalis en el material crudo frecuentemente se encuentren en forma de feldespatos insolubles o formando otros silicatos, sin embargo, en la mayor parte, se convierten en sulfatos solubles durante el proceso. de cocción. Los intentos para reducir a pasta este polvo rico en sulfatos han dado como resultado masas viscosas no transportables por bombeo y que coagulaban cuando se introducían en el horno formando un anillo de fango. Los polvos con contenidos en sulfatos bajos no presentan estos inconvenientes, lo cual sugiere la idea de reducir los sulfatos alcalinos aun cuando no se requiera un cemento con bajo contenido en álcalis. Algunos operadores dicen que la lixiviación de los sulfatos alcalinos del polvo es el mejor medio para tratar los polvos que se han de retornar al horno. La Missouri Portland Cements Company recoge el polvo, lo mezcla con agua a razón de $1: 10$, en peso, y lo bombea a un pequeño espesador. El líquido completamente saturado de sulfatos alcalinos solubles se descarga, y la pasta depositada, que contiene un $50 \%$ de agua, se la retorna a un horno independiente mediante una bomba centrifuga de velocidad variable con carga uniforme de casi $3 \mathrm{t}$ por hora. Con este tratamiento se separa cerca del $90 \%$ de los sulfatos alcalinos solubles y produce deI 8 al $12 \%$ de la alimentación del homo casí exenta de álcalis.

Las condiciones necesarias para una lixiviación satisfactoria son: 1) Una relación de disolución del agua respecto al polvo superior a 8:1 para evitar la formación de costra; y 2) Agitación continua de la pasta, porque si se la deja depositar lentamente, como sucede en las conducciones de tuberías horizontales o en conductos cerrados, la pasta endurecerá fraguando. Otras ventajas que se obtendrán en el funcionamiento del horno son la reducción de la carga de polvo que circula por el precipitador, la reducción de la formación de costra en el precipitador, un mejor control del fraguado, posibilidad de utilizar carbón con alto contenido de azufre sin que, por ello, sea excesiva la absorción de sulfatos en el clínker, reducción de la carga circulante de azufre en el horno $\mathrm{y}$, en consecuencia, reducción de la formación de anillos de sulfatos antes de la zona de cocción y reducción de los anillos de pasta.

C. F. Clausen ha propuesto un sistema aplicable a los hornos de tipo Lepol con precalentador de parrilla móvil, mediante el cual los gases de los homos quedan completamente eliminados del sistema después de pasar a través de la sección de recalentamiento por la parrilla 
móvil. El aire caliente del enfriador se usa después para secar el granulado. Clausen cree que puede aplicarse el mismo principio a otros hornos, ya sean convencionales o de precalentador por suspensión. No se trata de aumentar la economía de combustible, sino, sobre todo, de eliminar álcalis.

Se ha mencionado que la difusión interna de los álcalis hacia la superficie de los granos de clínker representa un factor determinante en la limitación de la velocidad de volatilización. De este hecho puede conseguirse que los granos pequeños de clínker retendrán un porcentaje de álcalis más bajo que los grandes, lo cual se ha confirmado en algunos casos. Por consiguiente, un medio para reducir el contenido de álcalis será el de cambiar los procedimientos de elaboración para producir un clínker de granos más pequeños.

Además se ha hecho otra propuesta: la de reducir los álcalis introduciendo una pulverización de agua en la zona de cocción del horno. Ahora bien, los beneficios y eficacia de una y otra propuesta no han sido demostrados.

\section{El automatismo}

Automatismo es mucho más que instrumentación. El automatismo implica control automático, mientras que la instrumentación se limita a recurrir al uso de instrumentos en lugar de los ojos y las manos del hornero para prestar mayor atención a las alarmas. El control de cualquier proceso se realiza en tres fases: primero, un dispositivo de percepción que indica la nece. sidad de una variación; segundo, un dispositivo de «servo-comando» que efectúe las variaciones necesarias; tercero, un dispositivo de registro que demuestre el efecto de las variaciones. En el control de tipo tradicional el dispositivo de percepción es el ojo del hornero, el de comando es su mano, que acciona una válvula o un interruptor, y el de registro es un lápiz en su mano que anota el efecto.

Si la instalación del horno está parcialmente instrumentada, el hornero puede ayudarse para sus apreciaciones de los diversos instrumentos: los pirómetros pueden indicar e, incidentalmente, registrar la temperatura de la zona de cocción o de la zona de alimentación; otros aparatos de medida pueden indicar y, eventualmente, registrar la presión y la cantidad de gas; los analizadores de gas de escape pueden indicar y, casualmente, registrar la composición de la atmósfera del horno. Pero únicamente el hornero es el responsable tanto del control de los indicadores y de los gráficos, así como de la realización, por sus propios medios, de las maniobras que puedan producir el cambio que él desea hacer.

EI progreso obtenido con el automatismo estriba en el hecho de que el dispositivo de percepción acciona un relé un cierto tiempo, que transmite el comando a la unidad por medio del cual se realiza automáticamente la variación, exactamente en la medida requerida. Cualquier actuación se registra continuamente para las sucesivas observaciones. La gran diferencia entre el control manual y el automático es que el hombre siente la necesidad sólo de grandes cambios, lo cual requiere grandes correcciones a largos intervalos, mientras que un instrumento puede percibir las mínimas variaciones y seguir pequeñ́simas correcciones en intervalos de tiempo brevísimos. El resultado en el lenguaje de los gráficos registrados será, en un caso, una curva muy desigual y, en el otro, una curva limpia. La marcha, en un caso, es irregular y costosa y produce un clínker de calidad variable; en el otro caso es uniforme y económica y produce un clinker de calidad óptima constante. Estas afirmaciones puede que sean exageradas en relación con la experiencia actual, pero los fabricantes que han adoptado el automatismo no hacen más que elogiarlo entusiásticamente.

El automatismo en la industria del cemento ha tenido un desarrollo gradual, pero muchos establecimientos parcialmente automatizados funcionan de forma muy satisfactoria. En Calaveras, los pirómetros de radiación fueron retirados del servicio por su inexactitud en la medida de las temperaturas debido al polvo en la atmósfera del horno. Pero en su lugar se instaló un tubito 
de $25 \mathrm{~cm}$ de diámetro y $30 \mathrm{~cm}$ de longitud, soldado a la parte externa del horno de $110 \mathrm{~m}$, en la parte correspondiente a la zona de cocción a $52 \mathrm{~m}$ de la boca de descarga. Un termopar inserto en el tubo, revestido con refractario, registra la temperatura, que varía en aquel punto entre $735^{\circ} \mathrm{C} \mathrm{y} 785^{\circ} \mathrm{C}$. El indicador de temperatura está conectado por medio de un relé a un regulador de carga, el cual dirige la posición de la váivula del gas de combustión. La carga de combustible regula la cantidad de aire primario, mediante variaciones adecuadas de velocidad que gobiernan la velocidad de giro del ventilador. La relación entre tal velocidad y la cantidad de los gases es también regulada por otro medio de control conectado a un analizador de oxígeno. Por tanto, en resumen, la temperatura de la carga próxima a la zona de sinterización regula el tiro del gas, que, a su vez, regula la velocidad del ventilador en una forma dirigida por el contenido de oxígeno de los gases de escape.

En la cementería de la Ideal Cement Company, en Tijeras, una unidad de televisión industrial montada en la cabeza de descarga permite al operario tener cuidado del horno y observar la marcha del clínker incandescente que se aproxima a la descarga. Este sistema es inusitado, en cuanto a la defensa de la TV, que está montada en una cámara de control con aire acondicionado, desde la cual se controlan todas las operaciones de la fábrica de cemento: desde la cantera a la sección de ensacado. Ün diagrama luminoso muestra todas las máquinas que se encuentran en funcionamiento. Dispositivos de seguridad y relé señalan las averías en cualquier punto del sistema y paran la maquinaria en caso de dificultad.

Otro paso hacia la completa automatización se ha llevado a cabo en la Riverside Cement Company, en su fábrica de cemento de Oro Grande, en California. Características particulares, aparte de la defensa de la TV de la zona de cocción, son: 1) Un calculador digital que, con una cantidad dada, una composición química determinada y señalando la localidad de origen de la caliza y de las otras materias primas, calcula las cantidades que deben añadirse de cada componente del depósito-hangar para obtener la dosificación deseada en la mezcla cruda; 2) Un sistema de tubos neumáticos que, en pocos segundos, transporte las muestras de materia prima desde donde se efectúa la toma al laboratorio, y 3) Un esfectrómetro de rayos $\mathrm{X}$ que analiza las muestras de materia prima. Basados en los datos facilitados por el calculador digital, se seleccionan los materiales y después se envían por medio de un transportador de cinta a otro transportador, móvil, de tipo especial. Este transportador se desplaza a lo largo de cualquier montón de $11 \mathrm{~m}$ de altura y $152 \mathrm{~m}$ de longitud distribuyendo los materiales en una serie de capas. Los materiales del apilamiento homogeneizado se extraen por medio de una draga de rueda de cangilones de tipo especial, la cual se mueve en el montón, arrastrando una sección transversal continua de materia prima mezclada.

Una toma de muestras adecuada de materiales de composición diversa es una necesidad elemental para una alimentación uniforme del horno. En Oro Grande, el material de la estación secundaria de trituración tiene un dispositivo que verifica la toma de muestras continua para hacer un control previo antes de que entre dicho material en los mezcladores y en la zona de almacenamiento. Un aparato tomamuestras se desplaza en diversas direcciones a través de los materiales que deposita cada alimentador rotatorio que alimenta a un molino de bolas ventilado. Los finos del molino se arrastran a través de un ciclón y se descargan en un segundo aparato tomamuestras, con el que se obtiene una muestra de crudo perfectamente homogeneizada.

El programa lineal es dado por un calculador R'W 300 (de la Thompson Ramo 'Wooldridge Products Company), mediante el cual se obtiene la combinación de más bajo costo con las materias primas disponibles para obtener una mezcla de composición química determinada. La composición deseada puede expresarse en función de los óxidos, de los compuestos, de los módulos o de combinaciones de estos factores. Dadas ocho materias primas diferentes y un crudo determinado, expresado en porcentajes de $\mathrm{SiO}, \mathrm{Al}_{2} \mathrm{O} ., \mathrm{CaO}, \mathrm{MgO}$ y álcalis, el calculador tarda de 10 a $30 \mathrm{mn}$ para resolver el problema, mientras que una persona provista de tablas de cálculo empleaba varios días. Las informaciones o datos necesarios comprenden análisis completos y datos de todos los depósitos de materia prima y de los costos unitarios. 
Se han hecho grandes progresos en el análisis rápido con la espectrografía por rayos $\mathrm{X}$, pero esta innovación no se ha desarrollado todavía por completo. Los instrumentos son extremadamente sensibles a muchas variables, tales como la concentración, hábito cristalino, finura y distribución granulométrica, técnica de preparación de las muestras y otras condiciones no definidas. Por otra parte, el aparato de rayos $\mathrm{X}$ no es totalmente estable $\mathrm{y}$, por tanto, la respuesta puede ser significativamente variable de un día para otro. Las muestras standard deben ser del mismo origen que las examinadas, molidas de la misma manera, con la misma finura y recogidas en los mismos recipientes y con la misma presión. Pero las dificultades se van superando con una experiencia cada vez mayor. Si se trabaja bien, el método es rápido y de toda confianza.

El automatismo en las fábricas de cemento del futuro deberá tener en cuenta los datos del mercado, la producción, la marcha del tiempo, etc., con los costos y con las ventas, lo cual determinará la política de la Sociedad. Esta establecerá la fe del personal en la maquinaria que se les confía y fijará la economía de las diversas operaciones parangonando un procedimiento con otro, una fuente de materia prima con otra y también los diversos mercados entre sí.

\section{La investigación}

No puedo abandonar el argumento del desarrollo tecnológico sin mencionar el apoyo fundamental que ha hecho posible tal desarrollo: la investigación.

Aun cuando la población crezca y la sociedad se haga cada vez más compleja y los recursos naturales disminuyan, nosotros dependeremos cada vez más de la ciencia y de la tecnología. Cada vez con más intensidad la ciencia y la tecnología intervienen en los procesos proporcionando las soluciones fundamentales.

El Dr. Brode, en su primera alocución ante el IV Congreso Internacional de la Química del Cemento, exponía algunos datos estadísticos muy significativos. Dice que hay un aumento del $10 \%$ anual en nuestra literatura científica, que equivale a duplicarla al cabo de ocho años, frente a un aumento de un $1,7 \%$ de la población mundial, que se duplica cada cincuenta años.

En los últimos diez años se ha publicado tanto como en el período precedente desde el descubrimiento de la imprenta, y hoy día viven el $90 \%$ de todos los científicos en él comprendidos.

En el año 1950 la industria americana gastó 8.200 millones de dólares en la investigación $\mathrm{y}$ en estudios financiando un $56 \%$ el Gobierno Federal. Esta es la mayor suma que se ha gastado en el mundo para investigaciones y estudios en todos los tiempos hasta el año 1950.

El dominio del hombre sobre la naturaleza, sobre sus fuerzas y sobre sus materias primas es el factor más potente que determina el modo de vivir, la economía, el nivel de vida, la religión.

Las nuevas industrias creadas en un país se convierten en centros de nuevas comunidades, absorbiendo desocupados y procurando nuevos medios para hacer la vida más agradable. Todo esto es fruto de la investigación y del estudio.

La industria del cemento no es una excepción en lo que se refiere a beneficios obtenidos mediante la investigación.

En la primera mitad de este siglo, la finalidad de un laboratorio para el cemento era la de experimentar las características de clinkerización de un gran núcleo de combinaciones con materias primas y la de poder correlacionar los cementos que resultaban con las propiedades de los hormigones obtenidos con los mismos. 
Durante el primer cuarto del presente siglo se recogieron numerosísimos datos para establecer métodos de ensayo para la normalización y la dosificación del hormigón.

La investigación se orientó en su campo específico en el segundo cuarto de siglo; es cuando los químicos y los físicos, a través de los laboratorios dedicados al cemento en todo el mundo aplicaron las leyes de la ciencia al arte de producir cemento y a su utilización.

El éxito de su esfuerzo ha sido la enorme expansión de la industria a raíz de las directrices que quedaron marcadas y estimuladas como consecuencia de los nuevos conocimientos científicos.

Durante los últimos años los progresos han sido más rápidos, lo que parece indicar la iniciación de una nueva era en el pensamiento científico de la industria.

El investigador tiene nuevos instrumentos para explorar Ia estructura molecular y cristalográfica de las fases que constituyen el cemento y sus productos de hidratación. La importancia de estas estructuras se explica con el carácter de las informaciones que se pueden obtener de su perfecto conocimiento.

El productor dispone así de nuevos medios de control para la producción y para la calidad de su producto, que le permiten adaptarse a las necesidades, cada vez mayores, de la industria de la construcción; por otra parte, la ingeniería civil dispone ahora de nuevos medios de control para la dosificación y economía de su hormigón con el fin de adaptarse a cualquier combinación de la demanda comercial.

Aun cuando se han hecho grandes progresos en el conocimiento de las leyes fundamentales de la física y de la química de los materiales conglomerantes, existen todavía grandes lagunas en el conocimiento de la morfología e identidad de las fases (inicial, intermedia y final) que se forman durante la hidratación y fraguado del cemento portland.

Los más importantes entre los nuevos medios de investigación en este campo-ya dados a conocer en el Simposium de Wáshington de 1960-son: los basados en la difracción de los rayos X; en la difracción electrónica, y en el microscopio electrónico.

Los cristales están compuestos por átomos que ordinariamente forman una celda elemental que se repite hasta la formación de un gránulo.

El comportamiento de una sustancia viene determinado no sólo por la naturaleza y la concentración de los átomos, sino también por la disposición de los átomos en el retículo; de aquí que el conocimiento y la disposición de los átomos en la célula elemental pone a nuestra disposición una explicación de las propiedades de una sustancia; esto nos muestra el porqué la sustancia tiene las propiedades que le son características.

Por ejemplo: tenemos diversas formas cristalinas del silicato bicálcico; sabemos que una de éstas, la forma $\gamma$, no reacciona con el agua, y por consiguiente, no es deseable en el clínker. Otra forma, la $\beta$, reacciona lentamente con el agua y constituye normalmente un notable porcentaje del clínker de cemento. La pulverización espontánea del clínker es debida a la inversión de la forma $\beta$ a la $\gamma$.

Pero el conocimiento de la disposición del átomo en la celda elemental indica también los modos mediante los cuales un determinado retículo puede modificarse, y los medios y la energía que se debe disponer para obtener la modificación deseada.

Mediante la aplicación de tal principio científico se han sintetizado diversos minerales y se ha desarrollado un control sistemático de sus propiedades. Así, la presencia de componentes menores-mineralizantes, gases, cenizas de carbón, etc--tiene un significado muy distinto de aquel que relaciona únicamente su efecto parcial con la composición total. 
Las reacciones, no sólo de la formación del clínker, sino también las de hidratación del cemento, parecen depender de la energía interna y de la estructura de los sólidos en reacción.

La causa de una reacción no puede ser definida exclusivamente por la relación temperatura/ concentración, sino que debe relacionarse también con los datos de sus niveles energéticos, estados reticulares, reacciones y transformaciones casuales.

Puesto que tales factores son susceptibles de regulación, el operador tiene en su propia mano un nuevo y potente medio para el control integral del proceso.

Solamente en los últimos años es cuando la técnica de la cristalografía por rayos X, combinada con el perfeccionamiento de los calculadores electrónicos, han hecho práctico este método para la determinación de la estructura cristalina.

Este método es todavía nuevo en los laboratorios de investigación del cemento, pero ya se está utilizando intensamente en muchos países.

Parte fundamental de tal programa es la determinación, mediante difracción por rayos $\mathrm{X}$, de la estructura cristalina de los compuestos del cemento.

También cabe esperar que exista un paralelismo entre la disposición de los átomos en la estructura cristalina, que puede ser determinado con toda exactitud, y su disposición en las fases amorfas, que a veces no puede determinarse con claridad.

Yo pienso, en consecuencia, que el conocimiento de la estructura de los compuestos puros bien cristalizados será directamente aplicable al estudio de las fases impuras mal cristalizadas o amorfas del clínker de cemento y, además, será también aplicable a la estructura del «gel» en la pasta de cemento.

La reactividad química de una determinada sustancia es función de la fuerza con que sus átomos están enlazados; la distancia interatómica, que puede determinarse mediante el análisis de la estructura cristalina, es una indicación de la fuerza de los enlaces químicos.

Para emprender el análisis estructural de un compuesto dado es necesario ante todo encontrar el camino para producir cristales del compuesto que sean ligeramente más grandes que. las dimensiones microscópicas.

Para lograr esta finalidad hemos consiruido un aparato de filamento caliente, para observar, con un microscopio de baja potencia, el comportamiento de pequeñas muestras a alta temperatura exactamente controladas. Así hemos observado directamente, en un reducido campo, muchos de los fenómenos que tienen Iugar en el horno.

El microscopio electrónico y la cámara de difracción electrónica son capaces de proporcionarnos resultados maravillosos.

La vista es el mejor medio de información que tienen nuestros sentidos, y cualquier cosa que amplíe su campo de empleo pone a nuestra disposición nuevos conocimientos científicos.

El microscopio electrónico proporciona los medios para la determinación visual de las dimensiones, forma y estructura de las partículas del gel, de las fases cristalinas que se forman durante la hidratación del cemento y también de las fases del clínker. Con su poder resolutivo amplía el campo visual a más de veinte unidades Amgstron (unas 0,002 micras), cien veces más que un microscopio óptico. Su utilidad es tal que la imagen puede ser ampliada hasta cien mil diámetros.

Además, puede emplearse un método conocido con el nombre de «téplica» o «sombra», mediante el cual una pátina de un metal especial vaporizado se proyecta sobre un objeto y se 
obtiene una imagen que parece ser tridimensional. De esta forma puede medirse la altura del objeto siendo posible distinguir, por ejemplo, con seguridad, una esfera de un disco.

La cámara de difracción electrónica es similar al microscopio, salvo que la disposición de los lentes permite la transmisión de los espectros de difracción, en lugar de la imagen reconstruida. Esto permite grandes ventajas especiales para identificar la naturaleza cristalina de superficies, películas o fibras, pudiéndose realizar en cantidades o dimensiones muy pequeñas para el análisis difractométrico con rayos $\mathrm{X}$.

Puesto que los productos principales de la hidratación del cemento portland son de dimensiones coloidales, este instrumento posee ventajas únicas para este empleo.

Estos dos instrumentos suministran los medios para delinear y resolver la microestructura de los productos de hidratación del cemento que no se pueden determinar con otros medios.

Las posibilidades, por otra parte, no están ausentes de limitaciones y restricciones, puesto que para estos instrumentos tan extraordinarios deben desarrollarse técnicas especiales e interpretaciones adecuadas.

Un perfeccionamiento reciente de gran valor es el contituido por el selector de fases, mediante el cual una partícula observada al microscopio puede aislarse de su campo y utilizarla para obtener un espectro de difracción. Conviene señalar, por otra parte, que pueden alcanzarse conclusiones válidas sólo basándose en datos concordantes sacados de experimentos repetidos sistemáticamente realizados para producir una familiarización y una confianza en las observaciones y a la Iuz de las teorías existentes y de otros numerosos datos obtenidos independientemente con el uso de otros instrumentos y métodos.

Citaremos, por ejemplo, la técnica de la difracción con bajo ángulo, que es de gran valor para confirmar las dimensiones de las partículas muy pequeñas observadas en el microscopio electrónico.

Puesto que estos instrumentos son relativamente nuevos, el campo de la investigación sobre el cemento, con su empleo, es todavia más amplio.

Los estudios que parece urgente anteponer en la investigación son los relativos a resolver la estructura cristalina de la mayor parte de las fases y de los compuestos del cemento, la naturaleza de los productos de hidratación, las reacciones de hidratación y de hidrólisis, el polimorfismo a alta temperatura de algunos de los compuestos del cemento, tales como el silicato bicálcico y el silicato tricálcico y las diferentes series de soluciones sólidas como la del sistema cal-alúmina-óxido de hierro.

Las técnicas de la difracción electrónica requieren perfeccionamientos, y tal medio podrá encontrar aplicación en el análisis estructural especialmente con películas muy delgadas y fibras que sean transparentes a los rayos $\mathrm{X}$.

He referido, dentro del tiempo disponible, y sólo brevemente, algunos de los grandes progresos que recientemente se han hecho en la tecnología del cemento, y sus métodos de investigación que constituyen lo más sobresaliente en el progreso tecnológico.

Hemos visto solamente el principio de un gran desarrollo de los nuevos conocimientos científicos, y sus efectos sobre la sociedad actual, pero nosotros estamos dentro y formamos parte de la misma.

Yo me limito a desear, como ya lo he hecho en ocasiones parecidas, que mediante nuestros esfuerzos y muestra visión puede surgir un mundo mejor y que la paz reine en todos los pueblos de la tierra. 